\title{
Diacronie
}

Studi di Storia Contemporanea

$N^{\circ} 8,4 \mid 2011$

Le sembianze di Clio: approcci alla storia

\section{Gabriele Balbi, Le origini del telefono in Italia. Politica, economia, tecnologia, società}

\section{Luca Zuccolo}

\section{Q OpenEdition}

Edizione digitale

URL: http://journals.openedition.org/diacronie/3494

DOI: 10.4000/diacronie.3494

ISSN: 2038-0925

Editore

Association culturelle Diacronie

Notizia bibliografica digitale

Luca Zuccolo, "Gabriele Balbi, Le origini del telefono in Italia. Politica, economia, tecnologia, società », Diacronie [Online], № 8, 4 | 2011, documento 11, Messo online il 29 octobre 2011, consultato il 23 septembre 2020. URL : http://journals.openedition.org/diacronie/3494 ; DOI : https://doi.org/10.4000/ diacronie.3494 


\section{RECENSIONE:}

\section{Gabriele BALBI, Le origini del telefono in Italia. Politica, economia, tecnologia, società, Milano, Bruno Mondadori, 2011, 226 pp.}

a cura di Luca ZUCCOLO *

Gabriele Balbi, ricercatore in Storia e teoria della comunicazione presso l'istituto di media e giornalismo dell'Università della Svizzera Italiana di Lugano, visiting scholar presso la Columbia University di New York e già autore di La Radio prima della Radio. L'Araldo Telefonico e l'invenzione del broadcasting in Italia (Roma, Bulzoni 2010), propone in questo saggio un interessante analisi delle prime fasi dello sviluppo del telefono in Italia.

Il testo qui presentato, tuttavia, non è un semplice studio sull'oggetto telefono in quanto tale, ma è un'indagine, redatta attraverso l'analisi di fonti inedite, sulle modalità di introduzione dello strumento e delle reti telefoniche nel nostro Paese e sui problemi che la telefonia italiana ha dovuto affrontare nei suoi primi quarant'anni di esistenza.

«In definitiva», riprendendo quanto sostiene l'autore nell'introduzione, «quella contenuta in questo libro non vuole essere solo una storia politica o economica o tecnica o degli utenti del telefono, ma vuole essere un'unica e multiforme storia di come la società italiana abbia metabolizzato il nuovo mezzo tra Otto e Novecento: per realizzare tale obiettivo ciascuna di queste quattro storie deve essere considerata allo stesso livello delle altre e, dalla loro reciproca influenza e contaminazione, possono emergere nuovi e fecondi aspetti della storia del medium» ${ }^{1}$.

${ }^{1}$ BALBI, Gabriele, Le origini del telefono in Italia. Politica, economia, tecnologia, società, Milano, Bruno Mondadori, 2011, p. 2. 
L'originalità di questa ricerca è insita innanzitutto nelle fonti utilizzate da Balbi, il quale sfrutta dei documenti fin'ora poco studiati, come i verbali dei dibatti parlamentari in materia di telecomunicazioni, i bollettini delle società telefoniche, le riviste specialistiche di elettrotecnica, tra cui spicca senza dubbio «L'elettricista», fino agli stessi elenchi telefonici che forniscono interessanti notizie sugli abbonati e sulla penetrazione sociale del mezzo telefonico.

Accanto alle fonti, tuttavia, anche la metodologia ha un ruolo fondamentale e innovativo, in quanto consente di «individuare alcuni passaggi inediti della storia del telefono (1880-1915 ndr) [...] allo scopo di arricchire il quadro di riferimento e di comprendere quella che può essere definita come la co-costruzione del nuovo mezzo di comunicazione ${ }^{2}$. Tra le teorie analitiche utilizzate da Balbi, accanto all'analisi storiografica, infatti, spiccano le tradizioni teoriche della Social Construction of Tecnology (SCOT), l'analisi economico-sociale delle telecomunicazioni e gli studi sui Macro Systèmes Tecnique (MST) e sui Large Technical Systems (LTS).

Il saggio si suddivide in sette capitoli, ripartiti cronologicamente secondo lo sviluppo storico della telefonia italiana dal 1877 al 1915-17. Nondimeno, l'aspetto cronologico serve solo da base per l'analisi di Balbi, che si sviluppa nell'intreccio delle dinamiche politiche, economiche, societarie, tecniche e sociali che hanno interessato il fenomeno telefonico in Italia. In ogni capitolo i dati e le contingenze storiche sono analizzate ed interpretate dai molteplici punti di vista dei soggetti sociali interessati. Pertanto, accanto alla descrizione delle leggi e delle loro ricadute pratiche, sono riportate le reazioni delle società telefoniche, dei tecnici e degli abbonati, categorie sociali diverse ma spesso sovrapposte o sovrapponibili che si palesano, nelle pagine di Balbi, come i veri attori del tormentato sviluppo delle origini della telefonia italiana.

Partendo da questa considerazione, si può osservare nel primo capitolo, che descrive il primo decennio dell'“era del telefono italiano”, dal 1877 al 1888, un'interessante analisi dei rapporti tra elettricità e telefonia, nonché le relazioni che intercorrono tra telegrafo e telefono. A questo riguardo è rilevante la parte dedicata ai primi rapporti tra politica e telefonia, e in particolare i lavori della commissione Tabarrini (1886), che per prima tentò una razionalizzazione del nuovo mezzo comunicativo con la già esistente rete telegrafica.

Accanto alla politica e ai suoi tentativi di regolamentare il nuovo strumento, tuttavia, va sottolineato anche l'emergere di un vivace settore privato, identificabile nel gran numero di società telefoniche nate in varie città per rispondere alla straordinaria "sete telefonica" degli italiani.

2 BALBI, Gabriele, Le origini del telefono in Italia, cit., p. 3. 
Balbi, infatti, in questo capitolo iniziale sottolinea più volte come il telefono sia stato ben accolto dal pubblico italiano, tanto da rendere il nostro Paese uno dei più all'avanguardia in Europa alla fine del secolo XIX. Tuttavia, questo fermento e questo desiderio di innovazione si contrapposero ad una situazione economico-politica molto ambigua e dominata dall'incertezza e dall'oscillazione tra il sistema pubblico e quello privato. Questo carattere di forte ambiguità è così forte che resterà la cifra dominante di tutto il periodo aurorale della telefonia italiana e verrà parzialmente risolto solo dopo la Grande Guerra.

A questo proposito è significativo il confronto, tracciato da Balbi nel secondo capitolo, tra la precoce nazionalizzazione europea e l'incertezza italiana, che vede, negli anni tra il 1888 e il 1900, l'aprirsi di una prima grande crisi della telefonia. Accanto all'incertezza sul sistema di gestione e alla pluralità di fornitori del servizio poco integrati tra di loro, si devono-aggiungere le resistenze del mondo politico e di alcuni governi al nuovo mezzo di comunicazione dovute ai pregressi ed ingenti investimenti telegrafici e alla scarsa stabilità dei governi in carica alla fine del secolo XIX. Questa situazione di stallo, in cui gli investimenti - sia pubblici che privati - scemarono per la scarsa possibilità di profitti, fu alla base della crisi dei telefoni, la quale fu aggravata dall'irrazionalità e dalla scarsissima diffusione della rete, sia urbana che interurbana.

La nascita e la difficile diffusione della rete interurbana viene trattata nel terzo capitolo in cui Balbi mette in evidenza le difficoltà incontrate da governo, società telefoniche e utenti nell'uso di questa rete tra il 1890 e il 1907.

Il quarto capitolo, al contrario, descrive i tentativi del governo di nazionalizzare il settore telefonico e lo scontro tra il mondo politico e le società private tra 1895 e 1907. In questo capitolo spicca il ruolo svolto dalla Banca Commerciale Italiana, che entra prepotentemente nel settore, e, in modo particolare, spiccano le prime reazioni dell'opinione pubblica e degli abbonati relativamente ai servizi telefonici offerti dalle compagnie concessionarie. In questo costante e continuo dialogo - che l'autore mette spesso in evidenza - tra governo, compagnie, tecnici e abbonati trova spazio anche la figura professionale dei centralinisti, o meglio, delle centraliniste: una figura professionale che la "sete telefonica" degli anni Ottanta dell'Ottocento ha fatto emergere nel contesto socio-economico italiano e che la crisi di fine XIX secolo ha portato alla ribalta per le dure condizioni di lavoro e per il nuovo rapporto sociale che si è venuto a creare tra utenti e centralinisti.

Dopo aver a lungo preparato il terreno, nel 1907 lo Stato italiano passa al riscatto e alla nazionalizzazione di parte delle linee telefoniche italiane. Questo è il tema del capitolo cinque, che si snoda tra le ragioni che hanno spinto il governo verso la 
nazionalizzazione e la dura critica operata da Saverio Nitti. Una dicotomia di pareri che mette, ancora una volta, in evidenza l'“esitanza naturale” tra pubblico e privato, caratteristica peculiare dell'approccio politico italiano alla telefonia.

Il capitolo sei-descrive il fallimento di questa prima e parziale nazionalizzazione dei telefoni italiani. Infatti, la rinnovata sete telefonica degli italiani, l'obsolescenza di molti impianti e reti telefoniche, unita agli scarsi investimenti e ad alcuni problemi strutturali e politici del nostro Paese, ha fatto letteralmente naufragare la nazionalizzazione, tanto che la gestione pubblica è andata subito in crisi e in deficit anche rispetto alle compagnie più piccole, rimaste ancora in mano ai privati, le quali anzi, conobbero un notevole sviluppo.

Col fallimento della nazionalizzazione si conclude la parte più tecnica del lavoro di Balbi, il quale nel settimo capitolo, dà spazio alla figura degli abbonati e alle ripercussioni che il telefono ha avuto sulla società italiana a cavallo tra XIX e XX secolo. In questa sede viene descritto il ruolo dei primi abbonati, la loro composizione sociale e professionale e, soprattutto, le loro reazioni alla penetrazione del mezzo telefonico nelle loro vite. L'avvento del telefono, infatti, è la causa di numerose "preoccupazioni sociali" che vanno dalla diffusione di malattie come la tubercolosi fino alla presenza dell'estraneo all'interno della propria casa e della propria intimità3 .

I primi quarant'anni della telefonia italiana sono dominati dall'incertezza, che si declina in varie forme, da quelle politiche a quelle economiche fino a quelle sociali, e questa indeterminatezza, citando ancora una volta Balbi, è forse una delle caratteristiche principali del contesto italiano. Durante questo periodo, infatti, le modalità d'uso, i significati, l'utilità, le potenzialità e la stessa struttura del nuovo medium non furono ben chiare; ciò contribuì a circondare il telefono di un alone di diffidenza e di incomprensibilità4.

Il saggio di Balbi è corredato e completato da un ottimo apparato di note, di tabelle e di grafici che aiutano il lettore ad orientarsi meglio nelle tematiche descritte e forniscono una prova tangibile dell'evoluzione materiale della telefonia italiana. La presenza di una dettagliata bibliografia, affiancata dal ricco elenco degli archivi consultati e da una serie di interessantissime appendici dona al saggio presentato un'ulteriore motivo di interesse e scientificità.

\footnotetext{
3 La presenza dell'altro e dell'estraneo, nonché il rapporto tra pubblico e privato nella società tardo ottocentesca e primo novecentesca hanno un grosso peso nell'immaginario collettivo e sociale egregiamente descritto dal sociologo Richard Sennett, in SENNETT, Richard, Il declino dell'uomo pubblico, Milano, Mondadori, 1982.

4 BALBI, Gabriele, Le origini del telefono in Italia, cit., p. 182.
} 
Il saggio,-sebbene specialistico è, al tempo stesso, un libro divulgativo, diretto anche ad un pubblico di non esperti del settore storico o delle comunicazioni. Il testo, scritto in un linguaggio chiaro e semplice, permette a tutti i lettori di comprendere le dinamiche storico-metodologiche alla base dell'aurorale sviluppo della tecnologia telefonica italiana. Il lavoro di Balbi, pertanto, è un utile strumento e un'ottima lettura per chiunque voglia comprendere la genesi della rete telefonica italiana e l'impatto sociale di questo rivoluzionario mezzo di comunicazione. 


\section{* L'autore}

Luca Zuccolo, dottorando (PhD Student) in Storia Contemporanea del SUM di Napoli sta sviluppando una ricerca sulla stampa francofona ottomana e la sua rappresentazione dell'Impero d'Oriente. Già dottore magistrale in Storia d'Europa (Bologna 2008), si è occupato della modernizzazione dell'Impero Ottomano, del confronto/scontro tra modernità e tradizione in questo contesto cosmopolita e dello sviluppo dei movimenti sociali che hanno preparato l'avvento della società turca contemporanea.

URL: < http://www.studistorici.com/2010/12/o1/luca-zuccolo >

\section{Per citare questo articolo:}

ZUCCOLO, Luca, «RECENSIONE: Gabriele BALBI, Le origini del telefono in Italia. Politica, economia, tecnologia, società, Milano, Bruno Mondadori, 2011, pp. 226», Diacronie. Studi di Storia Contemporanea: Le sembianze di Clio: approcci alla storia, 29/10/2011,

URL: <http://www.studistorici.com/2011/10/29/zuccolo_numero_8 >

\section{Diacronie Studi di Storia Contemporanea $\beta$ www.diacronie.it}

Risorsa digitale indipendente a carattere storiografico. Uscita trimestrale. redazione.diacronie@hotmail.it

Comitato di redazione: Marco Abram - Giampaolo Amodei - Jacopo Bassi - Luca Bufarale - Alessandro Cattunar - Alice De Rensis Barbara Galimberti - Deborah Paci - Fausto Pietrancosta - Martina Sanna - Matteo Tomasoni - Luca Zuccolo

Diritti: gli articoli di Diacronie. Studi di Storia Contemporanea sono pubblicati sotto licenza Creative Commons 2.5 Possono essere riprodotti a patto di non modificarne i contenuti e di non usarli per fini commerciali. La citazione di estratti è comunque sempre autorizzata, nei limiti previsti dalla legge. 